\title{
Using sparsity to estimate oscillatory mode from ambient data
}

\author{
SHEKHA RAI ${ }^{1, *_{0}}$, PRAVEEN TRIPATHY $^{2}$ and SISIR KUMAR NAYAK ${ }^{2}$ \\ ${ }^{1}$ Department of Electrical and Electronics Engineering, National Institute of Technology Nagaland, Nagaland, \\ India \\ ${ }^{2}$ Department of Electronics and Electrical Engineering, Indian Institute of Technology Guwahati, Guwahati, \\ India \\ e-mail: rai.shekha@gmail.com
}

MS received 27 March 2018; revised 21 June 2018; accepted 21 December 2018; published online 25 March 2019

\begin{abstract}
In this paper, a sparse-technique-based representation of the signal over a learned dictionary and random decrement technique are explored to extract the oscillatory mode from the ambient data. The main contribution of the present work is to design a dictionary and compute the coefficients that best represent the clean signal to estimate the modes. In this work, the noise embedded in the ambient signal is minimized by representing the ambient signal in sparse domain with respect to the dictionary. Comparison between the proposed method and other methods such as nonlinear filtering, etc., has been done on the test signal, two-area power system on the data generated through simulation in Matlab, two-area data simulated on real-time digital simulator and real measurement from Phasor data concentrator (PDC) of Indian power system and Western Electricity Coordinating Council (WECC) network.
\end{abstract}

Keywords. Inter-area oscillations; phasor measurement unit; wide area monitoring; sparse technique.

\section{Introduction}

The low-frequency oscillations of synchronous generators have emerged as one of the main factors that cause serious concern to the stability of power system. To ensure a secure and a reliable system operation, it is important to monitor these low-frequency modes in real time and observe that they have sufficient damping; in case the damping is poor, necessary action in the form of preventive control needs to be initiated. Fortunately, in the current years, with the rapid advancement of synchrophasor-based wide area measurement systems (WAMSs) that utilize GPS clock as time reference to estimate the phasors [1], the viability of capturing the real-time system dynamics has increased, thus enabling online monitoring and assessment of system stability. In India, establishment of WAMS and development of tools for real-time stability monitoring of Indian power system have been undertaken by the Ministry of Power, Government of India, from 2010 [2]. Synchronized measurements from phasor measurement units (PMUs) are used to estimate the presence of low-frequency oscillatory modes. The algorithms utilized for analysing these modes utilize either ringdown data or ambient data. The methods used for estimating the modes using ringdown data provide accurate estimation of modes present in the system with the occurrence of fault or some major disturbance, but as power

*For correspondence system keeps on changing its operating point, the estimated modes may not provide the current stability status of the system, whereas, the methods based on the uses of ambient data can provide the current status of the system.

Significant work has been done on the use of ringdown data for estimating the low-frequency mode such as the methods based on Kalman filter [3], Prony [4-6] and signal shift invariance techniques like TLS-ESPRIT [7]. However, the ringdown data are available only during the disturbance and the estimated modes provide the stability of the disturbed system, which may play a crucial role in mitigating the effect of disturbance in near future through online control, whereas the class of algorithms based on the use of ambient data provides the venerability of the system to the disturbance. The work related to the use of ambient data for mode estimation is like the methods based on the use of Yule-Walker method [8] for estimating the oscillatory modes, a stochastic-subspace-based method [9] and distributed frequency domain algorithm [10]. In the literature, random decrement technique (RDT) method with spectral independent component, wavelets and Ibrahim time domain (ITD) [11] has been cited in [12, 13] and [14], respectively. Reference [15] presents a comparative review of $[9,13]$ and [12]. In [16], a combination of eigen-realization algorithm (ERA) [18] and natural excitation technique (NExT) [17] has been used for detection of modes. RD-modified TLSESPRIT to mitigate the effects of nonlinear drifts has been cited in [19]. In [20] a wavelet-based method to derive a 
lower-dimensional measurement vector has been proposed, but requires a proper thresholding criterion for selection of significant wavelet coefficients, which to some extent utilizes a heuristic approach. Hence, there is an urge to develop an adequate method that can extract the signal components from the ambient data.

This paper utilizes the advantage of both sparsity and the RDT for estimating the oscillation modes of the ambient data. However, the primary focus of the paper is the investigation of the significance of sparse-representationbased methods [21, 22], which comprises designing an efficient dictionary from a given input signal and thereby extracting the representation coefficients from it that best represent the noise-free signal. Sparse representation over learned dictionary that has the capability to adapt to a given signal can capture the signal components more effectively. RDT is then used to get the free vibration response. The proposed method is compared to the nonlinear filtering, NExT-ERA, RDT-ITD and wavelet methods for the test signals, simulated second-order system corresponding to the modes of two-area system [23], two-area system simulated on real-time digital simulator (RTDS) facility at IIT Kanpur, real-time PMU data for Indian power systems and WECC $[24,25]$. The theoretical background of the techniques comprising the proposed method is presented in section 2 . Section 3 gives an outline of the use of proposed method for online estimation. Section 4 discusses the simulation results and lastly section 5 provides the conclusion.

\section{Techniques constituting the proposed method}

\subsection{Nonlinear filter}

Pre-filtering the nonlinear trends present in the ambient data obtained from the PMU is required. The mathematical equation corresponding to the nonlinear pre-filter [19] proposed in this work for removing the nonlinear trends is given by Eq. (1):

$$
\begin{aligned}
y[i] & =\frac{1}{2}\{(x[i]-\bar{w}(k-1))+(x[i]-\bar{w}(k))\} \\
& =x[i]-\frac{1}{2}\{\bar{w}(k-1)+\bar{w}(k)\} \quad \forall i=w_{s}, \ldots, \infty
\end{aligned}
$$

where $w(k)$ is the $k$ th window and $l_{w}$ is the length of the window $(\mathrm{W})$; the size of window in this work is always a multiple of $2 ; k$ is the index of the window, where $k=2, \ldots \infty ; w_{s}$ is the samples by which the window is shifted, which is given as $w_{s}=l_{w} / 2 ; x[i]$ is the input sample to the nonlinear pre-filter; $\bar{w}(k-1)$ is the mean of samples in $w(k-1) ; \bar{w}(k)$ is the mean of samples in $w(k)$ and $y[i]$ is the output of the nonlinear filtered signal.

It has been observed from the simulations that the nonlinear pre-filter has significantly mitigated the effect of the presence of nonlinear trends in the ambient data obtained from the PMU.

\subsection{Sparse technique for recovery of signal}

The signal after getting processed from the pre-filter [19] has very low SNR with very few modes present in the signal. Hence, to extract the signal, which lies in a verylow-dimensional space as compared with the dimension of the filtered ambient signal, the authors propose to use sparse-technique-based recovery of the signal. A brief introduction to sparse-technique-based recovery is provided in this sub-section.

Sparse representation [21, 22] of vector $\mathbf{x}$ is expressed by linearly combining selected atoms (columns) of the matrix D:

$$
\mathbf{x}=\mathbf{D} \gamma
$$

satisfying

$$
\min \|\mathbf{x}-\mathbf{D} \boldsymbol{\gamma}\|_{2}
$$

such that $\|\gamma\|_{1} \leq B$, where $\mathbf{D}$ is the dictionary and each column of the dictionary is called the atom. The vector $\gamma$ is called as sparse vector of dimension $D \times 1$, where some of its elements are non-zero and the remaining elements are zero. Total number of non-zero elements in $\gamma$, which is referred to as sparsity, is denoted by $B$. Dictionary $\mathbf{D}$ is created by exploiting the estimated structure of the signal using the measured data. The core idea of the sparse technique is to select the best possible combination of columns from the dictionary to represent the input signal. The main steps involved in the proposed method are (a) creation of the dictionary, (b) determination of sparse vector and (c) signal reconstruction, which are explained in the following sub-sections.

2.2a Creation of dictionary $(\boldsymbol{D})$ : The core idea behind the construction of the dictionary is to represent the signal with a minimum set of atoms from the dictionary and learn the dictionary that performs best on the signal. The efficiency of the proposed method greatly depends upon the creation of an effective dictionary $\mathbf{D}$ as the best combination of columns is chosen from the given dictionary to represent the clean signal; hence, the choice of a proper dictionary is very essential. In this work, a learned dictionary that offers flexibility and can fit the input signal better is created, which is discussed as follows. Data matrix $\mathbf{X}$, i.e., $\mathbf{X}=\left[\mathbf{x}_{\mathbf{0}} \mathbf{x}_{1} \ldots \mathbf{x}_{\mathbf{n}-1}\right]$, is created from the pre-filtered data using non-overlapping windows of samples, creating a column vector $\mathbf{x}_{\mathbf{i}}$ of length $l$ for the data matrix $\mathbf{X}$. The total number of samples utilized for mode estimation is, hence, given as $l \times n$. To get a low-rank approximation of the data matrix, singular value decomposition (SVD) along with the method proposed in [26] to get model order has been utilized. To get the model order, an index as proposed in [26] is defined in the following equation: 


$$
K(i)=\left[\frac{\rho_{1}^{2}+\rho_{2}^{2}+\cdots+\rho_{i}^{2}}{\rho_{1}^{2}+\rho_{2}^{2}+\cdots+\rho_{D}^{2}}\right]^{\frac{1}{2}}
$$

where index $K(i)$ increases monotonously and $i$ th singular value is denoted by $\rho_{i}$. When $i$ advances to the real order of the signal, $K(i)$ almost reaches 1 . The present work has utilized Eq. (4) to compute the order of the signal. After determination of the approximated low-rank data matrix, SVD is applied on it. The left singular vectors of the low rank data matrix form the dictionary for the proposed method. The atoms of the dictionary contain the significant eigenvectors corresponding to the signal subspace.

2.2b Computation of sparse vector $(\gamma)$ : Sparse vector $\gamma_{i}$ with respect to the dictionary $\mathbf{D}$ is determined for each column vector $\mathbf{x}_{\mathbf{i}}$ corresponding to the data matrix $\mathbf{X}=\left[\mathbf{x}_{\mathbf{0}} \mathbf{x}_{\mathbf{1}} \ldots \mathbf{x}_{\mathbf{n}-\mathbf{1}}\right]$ using a pursuit algorithm known as Batch OMP [27-29], which gives an approximate solution. Orthogonal matching pursuit (OMP) selects the atoms having the highest correlation to the current residual iteratively from the dictionary. However, it uses the current approximate of an orthogonal projection onto the subspace spanned by the selected columns thus far. Batch OMP performs faster than OMP as it involves computation of the projection of the residual onto the dictionary and not the residual itself.

The following terms used in the algorithm:

$n \rightarrow$ no of iteration

$I^{n} \rightarrow$ indices of the atom selected

$\gamma^{n} \rightarrow$ sparse representation of $\mathbf{x}$

$\mathbf{r}^{n} \rightarrow$ residue $\left(\mathbf{x}-\mathbf{D} \boldsymbol{\gamma}^{n}\right)$

$\zeta^{n} \rightarrow$ product of $\mathbf{D}^{T} \mathbf{r}^{n}$

$\mathbf{G} \rightarrow \mathbf{D}^{T} \mathbf{D}$

$\mathbf{Y}^{n} \rightarrow$ product of $\mathbf{G} \boldsymbol{\gamma}^{n}$

$\mathbf{L}^{n} \rightarrow$ Cholesky factorization of $\mathbf{G}_{I^{n}, I^{n}}$

$\mathbf{c}^{n} \rightarrow$ non-zero coefficients of $\gamma^{n}$, numbered by $I^{n}$

The computation steps are as follows [29].

1. Input: $\zeta^{0}=\mathbf{D}^{T} \mathbf{x}, \mathbf{G}$ and target sparsity $=B$

2. Output: The sparse vector $\gamma$

3. Initialization: Set $I:=(), \mathbf{L}^{1}:=[1], n:=1$

4. while $n \leqslant B$ do

5. $\hat{k}:=\underset{\mathrm{k}}{\operatorname{argmax}}\left\{\left|\zeta_{k}^{n-1}\right|\right\}$

6. $\mathbf{g}:=\mathbf{G}_{I^{n}, \hat{k}}$

7. if $n>1$ then

8. $\mathbf{w}:=$ solve for $\mathbf{w}\left\{\mathbf{L}^{n-1} \mathbf{w}=\mathbf{g}_{I_{n}}\right\}$

9. $\mathbf{L}^{n}:=\left[\begin{array}{cc}\mathbf{L}^{n-1} & 0 \\ \mathbf{w}^{T} & \sqrt{1-\mathbf{w}^{T} \mathbf{w}}\end{array}\right]$

10. end if

11. $I^{n}:=\left(I^{n-1}, \hat{k}\right)$

12. $\mathbf{c}^{n}:=$ solve for $\mathbf{c}\left\{\mathbf{L}^{n}\left(\mathbf{L}^{\mathbf{n}}\right)^{T} \mathbf{c}=\zeta_{I^{n}}^{0}\right\}$

13. $\mathbf{Y}^{n}=\mathbf{G}_{I^{n}} \mathbf{c}^{n}$
14. $\zeta^{n}:=\zeta^{0}-\mathbf{\Upsilon}^{n}$

15. $n=n+1$

16. end while

17. $\gamma:=0$

18. $\gamma_{I^{n}}:=\mathbf{c}^{n}$

Each sparse vector $\gamma_{i}$ corresponding to each of the column vector $\mathbf{x}_{\mathbf{i}}$ of the data matrix $\mathbf{X}=\left[\mathbf{x}_{\mathbf{0}} \mathbf{x}_{\mathbf{1}} \ldots \mathbf{x}_{\mathbf{n}-\mathbf{1}}\right]$ obtained through Batch OMP is arranged columnwise to get the resultant sparse matrix $\Phi=\left[\gamma_{0} \gamma_{1} \ldots \gamma_{\mathbf{n}-\mathbf{1}}\right]$.

2.2c Signal reconstruction: The dictionary $\mathbf{D}$ is multiplied with the estimated sparse matrix $\boldsymbol{\Phi}$ to get the reconstructed signal matrix $\mathbf{Y}_{\text {reconstruct }}$. The resultant signal vector $\mathbf{y}$ is obtained by appending all the columns of the reconstructed signal matrix. The reconstructed signal thus obtained represents a noise-free signal.

\section{$2.3 R D T$}

After extracting the signal components using sparse recovery technique, the next step is to derive the free vibration response of the system using a computationally fast timedomain averaging technique known as RD technique [30]. The signature for the random decrement method, which represents the free decay response, is estimated by taking the mean of the segments of fixed length from the signal. In the present work, the segments are picked once the signal exceeds a pre-determined level crossing threshold, which is taken as some percentage of the variance of the signal. The equation corresponding to RD signature is

$$
\delta(\tau)=\frac{1}{N} \sum_{j=1}^{N} y\left(t_{j}: t_{j}+\tau\right)
$$

where $N, \tau$ and $\delta(\tau)$ represent the segment number, length and RD signature, respectively. The length $\tau$ and threshold $T_{t h}$ must be selected properly for effective operation of the RD technique. ESPRIT-based method as proposed in [7] is then utilized to derive the low-frequency modes using $\delta(\tau)$ as input.

\section{Mode identification in power system utilizing the proposed method}

The block diagram in figure 1 depicts the step-by-step procedures for mode monitoring of power system in real time. Small and sudden mismatch between generated power and load demand results in the excitation of low-frequency modes, which are measured by the PMU. These measured data corresponding to the power swing are send to the PDC via the communication links. The proposed method firstly utilizes a block containing $N$ ambient samples. Then they are transformed in the sparse domain, which involves construction of a learned dictionary using SVD and computation of 


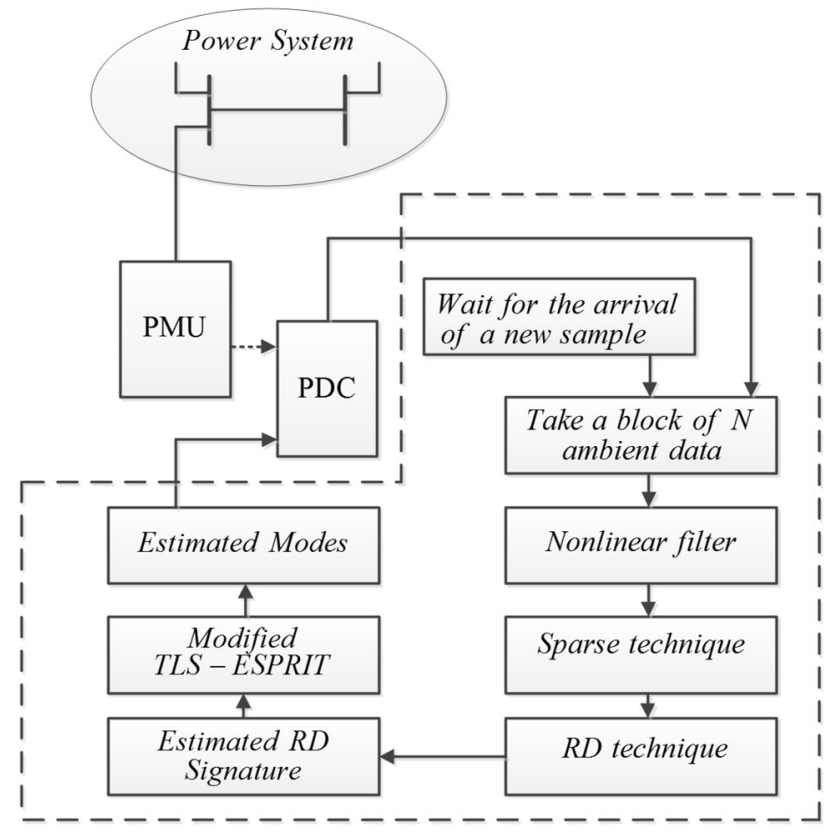

Figure 1. Block diagram of online mode estimation utilizing the proposed method.

representation coefficients using Batch OMP. Later, impulse response is obtained using RDT and finally the low-frequency oscillatory modes are estimated using modified TLS-ESPRIT.

\section{Simulation results}

For demonstrating the effectiveness of the proposed method, comparisons to nonlinear, NExT-ERA, RDT-ITD and wavelet methods are provided in the following subsection for the test signals, simulated second-order system corresponding to the modes present in a two-area system, two-area system simulated on RTDS facility at IIT Kanpur and real-time PMU data for Indian power systems and WECC. A performance identifier known as total vector error (TVE) [31] has been used for evaluating the accuracy of the proposed method:

$$
\operatorname{TVE}(\%)=
$$

where $X_{r}^{e}, X_{i}^{e}$ are the real and imaginary values of the estimated quantity and $X_{r}^{a}, X_{i}^{a}$ represent the real and imaginary values of the actual quantity, respectively.

\subsection{Mode estimation with simulated test signal resembling ambient data}

To generate the test signal resembling the ambient data, a second-order system with an attenuation factor $=-0.1$ and frequency $=0.2 \mathrm{~Hz}$ as shown in figure $2 \mathrm{a}$ is excited by Gaussian noise and corrupted with nonlinear trends. Finally, to get the test signal, the system's output response, shown in figure $2 b$, is sampled with sampling frequency $=$ $10 \mathrm{~Hz}$. The pre-processed signal is shown in figure 2c. Figure $2 \mathrm{~d}$ and e depicts the signal recovered using the sparse technique and the impulse response estimated by the RDT, respectively. Table 1 shows a performance comparison for all the methods. It has been observed that the proposed method estimates the modes more accurately (i.e., attenuation factor $=-0.0999$ and frequency $=0.1992 \mathrm{~Hz}$ ) with less TVE of $0.4025 \%$, in comparison with the other methods.

\subsection{Mode estimation for the two-area system}

To generate the test signal resembling the two-area ambient data, the authors have utilized two approaches, one by exciting the models of the second-order systems corresponding to the modes present in a two-area system and the other using random variation of load for a two-area system in RTDS.

4.2a Simulated two-area data: The ambient signal for a two-area power system is generated utilizing linear combination of 3 second-order models with Mode $1=$ $-0.25 \pm 7.5693 j$, Mode $2=-0.25 \pm 3.3765 j$ and Mode 3 $=-0.25 \pm 7.5084 j$ and nonlinearity generating functions as shown in figure $3 \mathrm{a}$. The output response as plotted in figure $3 \mathrm{~b}$ is sampled with sampling frequency of $10 \mathrm{~Hz}$ over 10-min time duration. The signal is preprocessed to obtain the filtered data and then it is reconstructed using sparse technique and finally RD technique is applied to estimate the RD signature. These data are used to show a comparison of the estimated dominant mode by the various methods and is listed in table 2 . It has been observed that the proposed method estimates the dominant mode more accurately (i.e., attenuation factor $=-0.2093$ and frequency $=0.5283 \mathrm{~Hz}$ ) with less TVE of $2.076 \%$. From the results presented in table 3 , it is seen that the proposed method outperforms the other methods in estimating the closely spaced modes with minimum TVE.

4.2b RTDS corresponding to two-area data: RTDS is used to generate real-time signals corresponding to interarea oscillations. Figure 4a shows the data that is generated for 20-min duration with sampling frequency of $50 \mathrm{~Hz}$, and the RTDS data corresponding to the low-frequency oscillations of the active power flow can be seen in figure $4 \mathrm{~b}$. The low-frequency oscillations have occurred due to random load changing in the range of $0-10 \mathrm{MW}$ at bus number 8 at an interval of $1 \mathrm{~s}$. It is seen from table 4 that the proposed method estimates mode more accurately (i.e., attenuation factor $=-0.2524$ and frequency $=0.5070 \mathrm{~Hz}$ ) with a TVE of $5.6416 \%$, which is less in comparison with the other methods. 


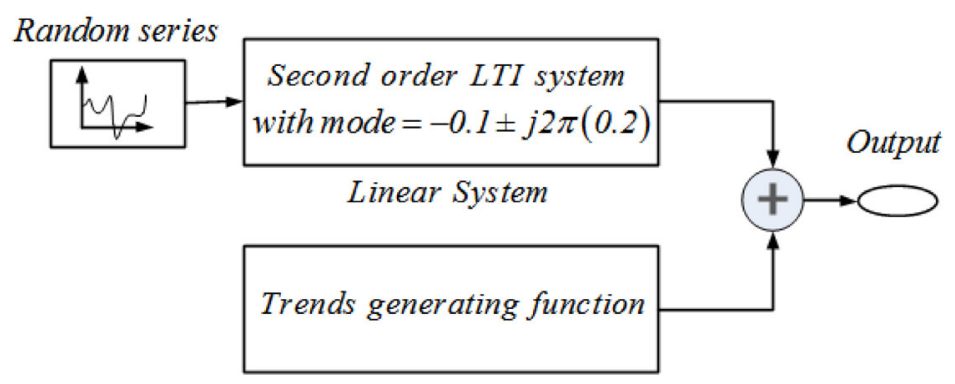

(a)

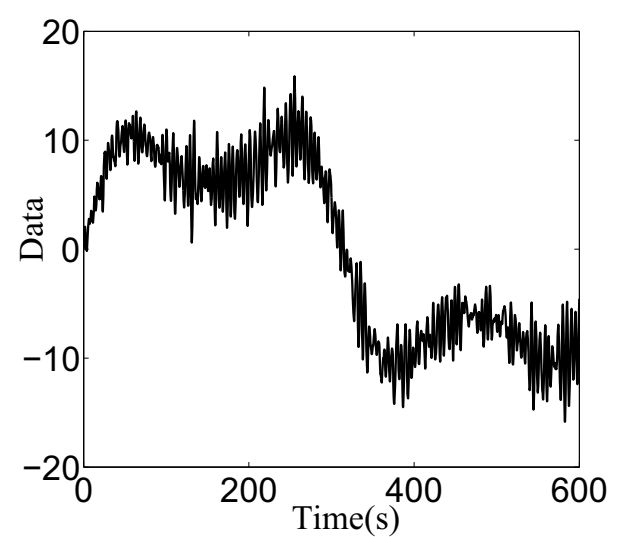

(b)

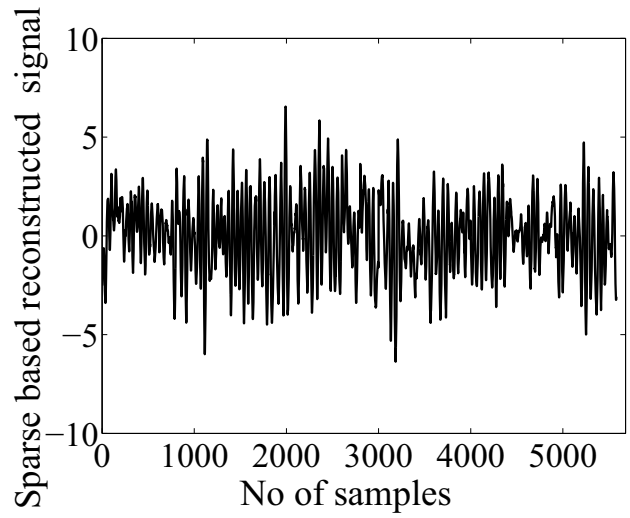

(d)

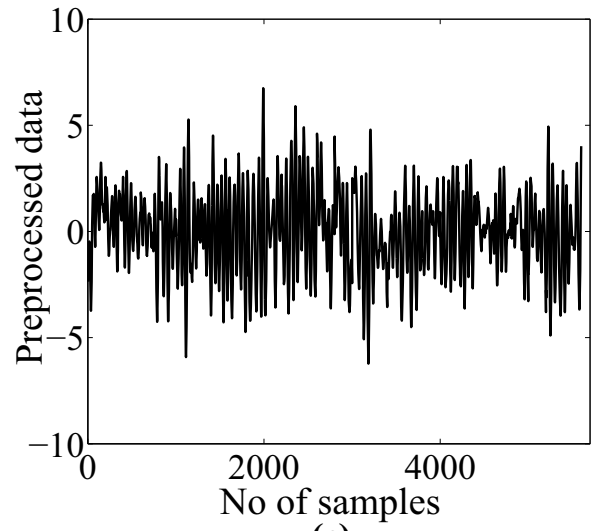

(c)

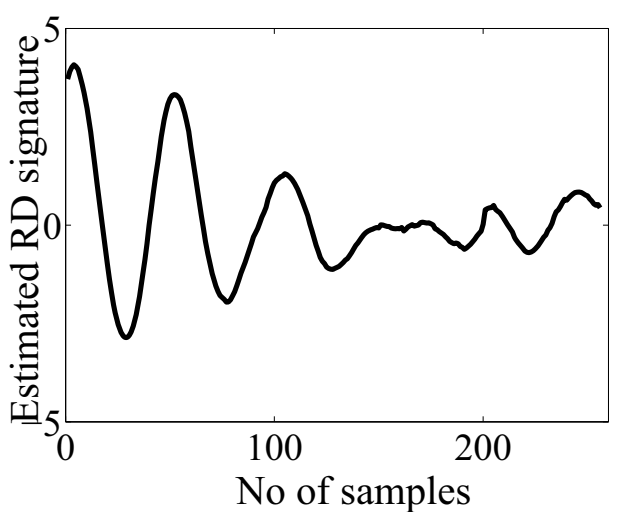

(e)

Figure 2. (a) SIMULINK model used to generate ambient signal; (b) generated test signal resembling ambient data corrupted with nonlinear trends; (c) preprocessed data; (d) sparse recovery signal and (e) estimated RD signature.

\subsection{Mode estimation utilizing the PMU connected at a bus in Indian power system}

To further illustrate the effectiveness of the proposed method, PMU data with phasor reporting rate of 25 frames/ second of North Eastern Regional Electricity Board (NEREB) of Indian power sector is used for mode estimation. The data measured from the PMU corresponding to the active power flow of the two lines incident on the same bus are provided in figure $5 \mathrm{a}$ and $\mathrm{b}$. Here, for calculating the TVE, we have assumed true value of the estimated modes as the average of the estimated modes for the two lines by the respective methods. 4.3a Active power flow data in the first line: Table 5 shows the mode estimated by all the methods for active power flow in the first line.

4.3b Active power flow data in the second line: Table 6 shows the mode estimated by all the methods for active power flow in the second line.

It is observed from tables 5 and 6 that the estimated modes for the data corresponding to the active power flow in both the lines, connected to a single bus, are more consistent for the proposed method in comparison with the other methods. The TVE for the proposed method provided in tables 5 and 6 is less in comparison with the other methods. 
Table 1. Estimated mode for generated test signal.

\begin{tabular}{lccc}
\hline Attenuation factor $=-0.1$, frequency & $=0.2 \mathrm{~Hz}$ & \\
\hline Methods & Est. atten. & Est. freq. & TVE $(\%)$ \\
\hline Nonlinear & -0.1033 & 0.1982 & 0.9258 \\
NExT-ERA & -0.1049 & 0.1970 & 1.5599 \\
RDT-ITD & -0.0870 & 0.2018 & 1.3554 \\
Wavelet & -0.0918 & 0.1981 & 1.1312 \\
Proposed method & -0.0990 & 0.1992 & 0.4025 \\
\hline
\end{tabular}

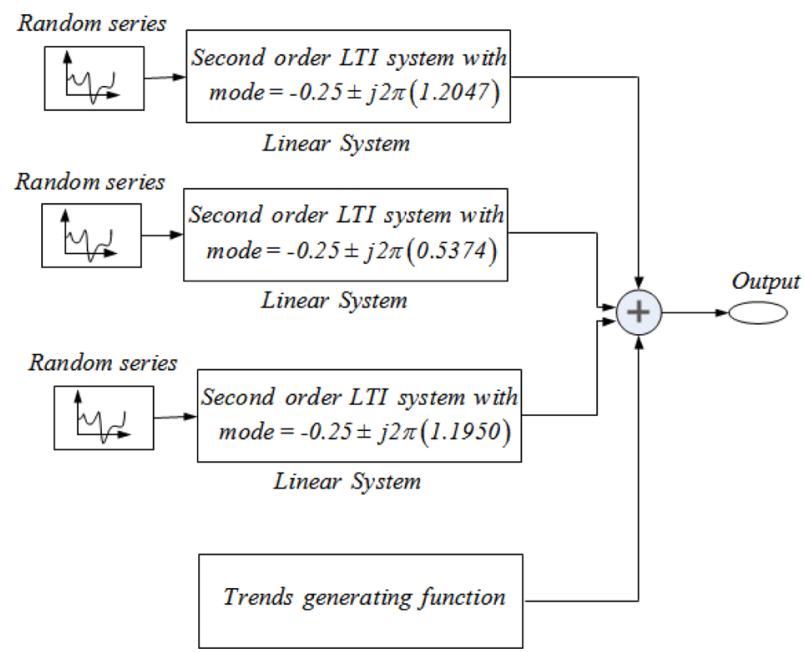

(a)

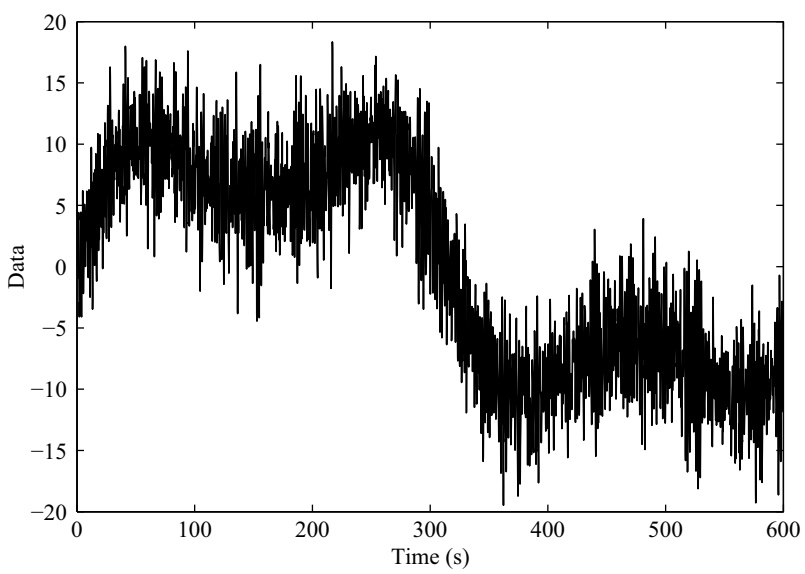

(b)

Figure 3. (a) SIMULINK model used to generate two-area data and (b) generated test signal resembling two-area data corrupted with nonlinear trends.

\subsection{Mode estimation utilizing the real probing data from the WECC system}

Probing data derived from the PMU connected to the WECC network, as shown in figure 6 , acquired on 14th
Table 2. Estimated dominant mode for generated two-area system.

\begin{tabular}{lccc}
\hline Attenuation factor $=-0.25$, frequency & $=0.5374 \mathrm{~Hz}$ & \\
\hline Methods & Est. atten. & Est. freq. & TVE $(\%)$ \\
\hline Nonlinear & -0.3188 & 0.5491 & 2.9724 \\
NExT-ERA & -0.2063 & 0.5216 & 3.1961 \\
RDT-ITD & -0.3152 & 0.5192 & 3.8954 \\
Wavelet & -0.3261 & 0.5469 & 2.8525 \\
Proposed method & -0.2093 & 0.5283 & 2.0760 \\
\hline
\end{tabular}

Table 3. Estimated modes for generated two-area system.

\begin{tabular}{lccc}
\hline Attenuation factor $=-0.25$, frequency & $0.5374 \mathrm{~Hz}$ & \\
\hline Methods & Est. atten. & Est. freq. & TVE $(\%)$ \\
\hline Nonlinear & -0.2054 & 0.5139 & 4.5512 \\
NExT-ERA & -0.2700 & 0.5224 & 2.8390 \\
RDT-ITD & -0.1167 & 0.5200 & 5.0901 \\
Wavelet & -0.2409 & 0.5130 & 4.5298 \\
Proposed method & -0.2721 & 0.5228 & 2.7879 \\
\hline Attenuation factor $=-0.25$, frequency & $1.2047 \mathrm{~Hz}$ & \\
\hline Methods & Est. atten. & Est. freq. & $T V E(\%)$ \\
\hline Nonlinear & -0.2482 & 1.2681 & 6.1176 \\
NExT-ERA & -0.1317 & 1.1700 & 3.2750 \\
RDT-ITD & -0.1766 & 1.2332 & 2.5831 \\
Wavelet & -0.1771 & 1.2976 & 7.7645 \\
Proposed method & -0.1846 & 1.2046 & 1.1865 \\
\hline Attenuation factor $=-0.25$, frequency & $=1.1950 \mathrm{~Hz}$ & \\
\hline Methods & Est. atten. & Est. freq. & $T V E(\%)$ \\
\hline Nonlinear & -0.0968 & 1.2569 & 4.7770 \\
NExT-ERA & -0.3438 & 1.1431 & 4.5189 \\
RDT-ITD & -0.2091 & 1.1265 & 5.7539 \\
Wavelet & -0.1142 & 1.1484 & 4.2951 \\
Proposed method & -0.1369 & 1.1925 & 1.8055 \\
\hline
\end{tabular}

September 2005 [25] have been utilized. The data used for the estimation purpose is taken from [24]. The modes estimated for the probing test signal were noted in [24] as $0.318 \mathrm{~Hz}$ with $8.3 \%$ damping.

Table 7 gives a comparison of all the mentioned methods for the probing test data. For the first window, the $\%$ damping estimated by the proposed method $(8.3896 \%)$ is almost equal to the value cited in [24]. The proposed method has been able to estimate the mode almost accurately $(0.2939 \mathrm{~Hz}$ at $7.87 .4 \%$ damping) with a minimum TVE $(7.6254 \%)$ in comparison with the other methods, for the second window. For window 1, the TVE for the estimated modes is comparable to those of the other methods. 


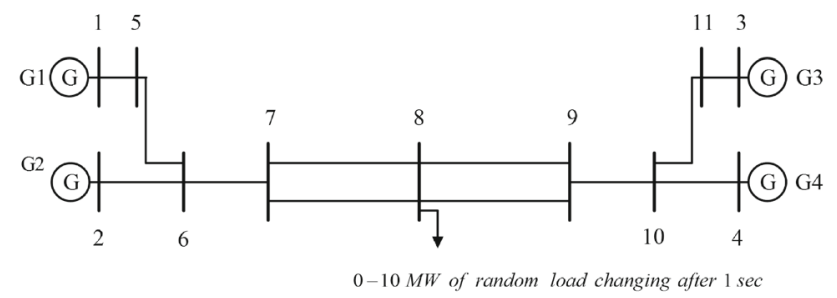

(a)

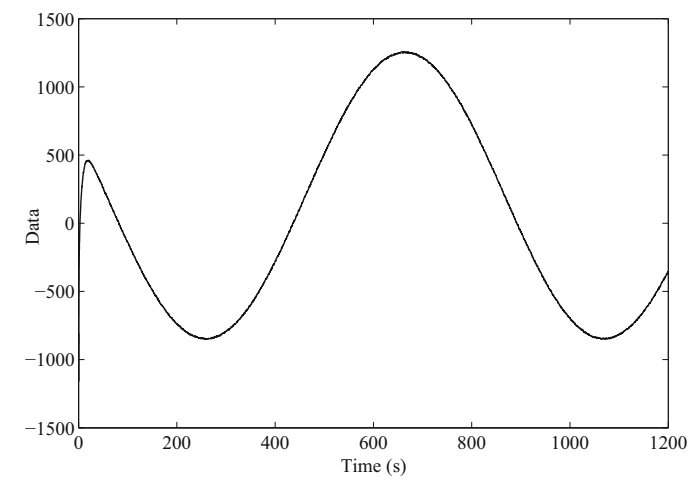

(b)

Figure 4. (a) Two-area system for ambient data and (b) RTDS data corresponding to active power flow of two-area system.

Table 4. Estimated mode with RTDS for two-area system.

\begin{tabular}{lccr}
\hline Attenuation factor $=-0.25$, frequency & $=0.5374 \mathrm{~Hz}$ & \\
\hline Methods & Est. atten. & Est. freq. & TVE $(\%)$ \\
\hline Nonlinear & -0.3410 & 0.2275 & 57.5793 \\
NExT-ERA & -0.2888 & 0.5025 & 6.5703 \\
RDT-ITD & -0.3393 & 0.7821 & 45.4787 \\
Wavelet & -0.1872 & 0.3947 & 26.5449 \\
Proposed method & -0.2524 & 0.5070 & 5.6416 \\
\hline
\end{tabular}

\section{Conclusion}

This paper presents a sparse-technique-based approach in which the ambient signal is expressed in the sparse domain with respect to the dictionaries, to extract the signal information. The proposed method designs a proper dictionary and chooses the optimum set of sparse coefficients to extract the clean signal, and hence it has significantly improved its robustness towards the presence of high level of noise and thus provides a more accurate estimation of the power system modes. To show its robustness, performance analysis of the proposed method with the nonlinear, NExTERA, RDT-ITD and wavelet methods is carried out for simulated signal, and for two-area system on the ambient data generated with MATLAB and RTDS. The efficiency of the proposed method is also elaborated on the real PMU data of NEREB and WECC system. From the results discussed in section 4 , it can be inferred that the sparse-

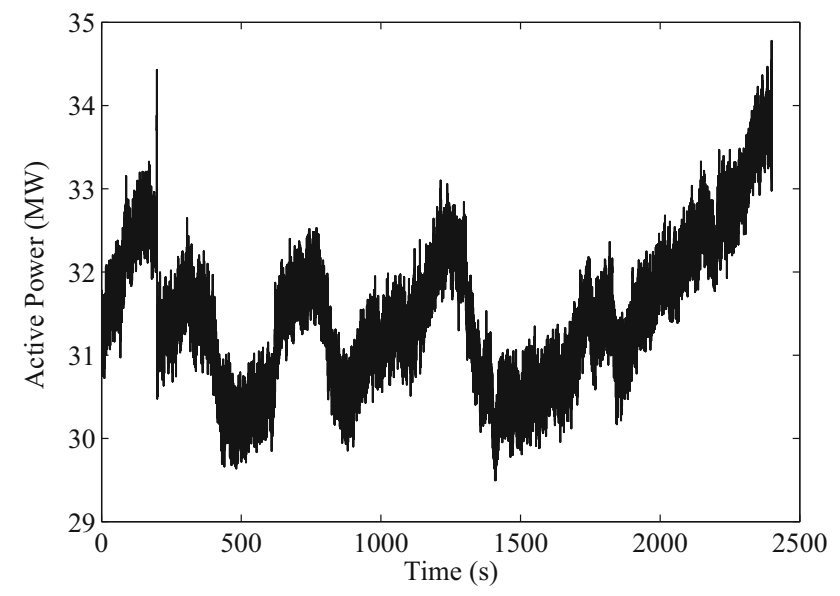

(a)

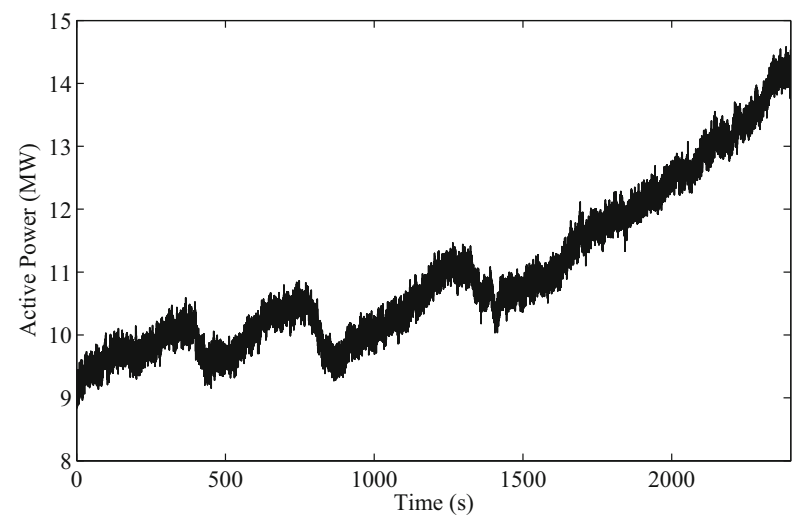

(b)

Figure 5. (a) Active power flow of the first line and (b) active power flow of the second line.

Table 5. Estimated mode for active power flow in the first line.

\begin{tabular}{lccr}
\hline Methods & Est. atten. & Est. freq. & TVE $(\%)$ \\
\hline Nonlinear & -0.4672 & 0.2193 & 24.2883 \\
NExT-ERA & -0.3938 & 0.2692 & 12.0351 \\
RDT-ITD & -0.4113 & 0.2250 & 19.3412 \\
Wavelet & -0.4629 & 0.2649 & 7.7845 \\
Proposed method & -0.4984 & 0.2342 & 7.2400 \\
\hline
\end{tabular}

Table 6. Estimated mode for active power flow in the second line.

\begin{tabular}{lccr}
\hline Methods & Est. atten. & Est. freq. & TVE $(\%)$ \\
\hline Nonlinear & -1.2741 & 0.2570 & 24.2899 \\
NExT-ERA & -0.8004 & 0.2526 & 12.0266 \\
RDT-ITD & -1.0342 & 0.2468 & 19.3407 \\
Wavelet & -0.7066 & 0.2884 & 7.7554 \\
Proposed method & -0.7291 & 0.2341 & 7.2347 \\
\hline
\end{tabular}

technique-based representation over dictionaries that contain the signal components can provide a more precise estimation of low-frequency oscillatory modes. 


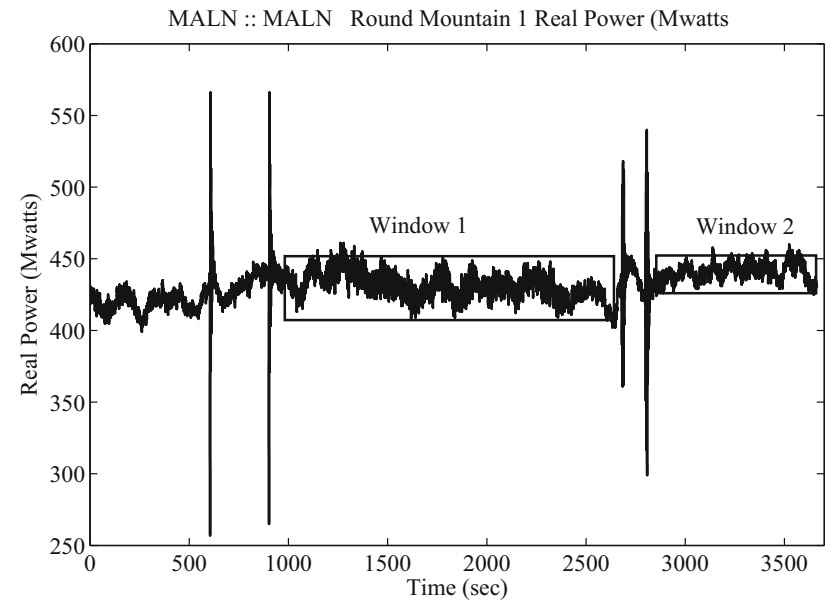

Figure 6. Probing data of WECC.

Table 7. Estimated mode for probing data of WECC.

Estimated mode for the first window

\begin{tabular}{lcccc}
\hline Methods & Est. atten. & Est. freq. & Damp (\%) & TVE (\%) \\
\hline Nonlinear & -0.1704 & 0.3139 & 8.6056 & 1.2982 \\
NExT-ERA & -0.1320 & 0.3193 & 6.5646 & 1.7639 \\
RDT-ITD & -0.1155 & 0.3286 & 5.5846 & 4.1717 \\
Wavelet & -0.1827 & 0.3107 & 9.3159 & 2.4150 \\
Proposed method & -0.1646 & 0.3111 & 8.3896 & 2.1607 \\
\hline
\end{tabular}

Estimated mode for the second window

\begin{tabular}{lcccc}
\hline Methods & Est. atten. & Est. freq. & Damp (\%) & TVE (\%) \\
\hline Nonlinear & -0.2607 & 0.2547 & 16.0807 & 20.3948 \\
NExT-ERA & -0.2407 & 0.2928 & 12.9698 & 8.7112 \\
RDT-ITD & -0.1210 & 0.2736 & 7.0249 & 14.1090 \\
Wavelet & -0.1656 & 0.2930 & 8.9586 & 7.8348 \\
Proposed method & -0.1458 & 0.2939 & 7.8704 & 7.6254 \\
\hline
\end{tabular}

\section{List of symbols}

\begin{tabular}{|c|c|}
\hline$w(k)$ & $k$ th window \\
\hline$l_{w}$ & $\begin{array}{l}\text { length of the window and must be a multiple } \\
\text { of } 2\end{array}$ \\
\hline$k$ & index of the window \\
\hline$w_{s}$ & samples by which the window is shifted \\
\hline$x[i]$ & input sample to the pre-filter \\
\hline $\bar{w}(k-1)$ & mean of samples in window $w(k-1)$ \\
\hline $\bar{w}(k)$ & mean of samples in window $w(k)$ \\
\hline$y[i]$ & output of the nonlinear filtered signal \\
\hline$\rho_{i}$ & $i$ th singular value \\
\hline$K(i)$ & monotonously increasing index \\
\hline$\gamma$ & sparse vector \\
\hline$B$ & $\begin{array}{l}\text { number of non-zero elements in the sparse } \\
\text { vector } \gamma\end{array}$ \\
\hline & number of iteration \\
\hline
\end{tabular}

$I^{n}$
$\gamma^{n}$
$\mathbf{r}^{n}$
$\zeta^{n}$
$\mathbf{G}$
$\mathbf{Y}^{n}$
$\mathbf{L}^{n}$
$\mathbf{c}^{n}$
$\mathbf{\Phi}$
$\mathbf{Y}_{\text {reconstruct }}$
$\tau$
$N$
$\delta(\tau)$
$T_{t h}$
$X_{r}^{e}$
$X_{i}^{e}$
$X_{r}^{a}$
$X_{i}^{a}$

indices of the atom selected sparse representation of $\mathbf{x}$ residue $\left(\mathbf{x}-\mathbf{D} \boldsymbol{\gamma}^{n}\right)$

product $\mathbf{D}^{T} \mathbf{r}^{n}$

product $\mathbf{D}^{T} \mathbf{D}$

product $\mathbf{G} \boldsymbol{\gamma}^{n}$

Cholesky factorization of $\mathbf{G}_{I^{n}, I^{n}}$ non-zero coefficients of $\gamma^{n}$, numbered by $I^{n}$ estimated sparse matrix reconstructed signal matrix segment length segment number RD signature threshold level for RDT real values of the estimated quantity imaginary values of the estimated quantity real values of the actual quantity imaginary values of the actual quantity

\section{References}

[1] Phadke A G and Thorp J S 2008 Synchronized phasor measurements and their applications. New York, Springer

[2] Sharma R B and Dhole G M 2016 Synchrophasor measurement network and its applications in Indian grid. In: Proceedings of the International Conference on Emerging Trends in Electrical Electronics Sustainable Energy Systems (ICETEESES 2016), Sultanpur, India, pp. 30-34

[3] Korba P, Larsson M and Rehtanz C 2003 Detection of oscillations in power systems using Kalman filtering techniques. In: Proceedings of the IEEE Control Applications, Istanbul, Turkey, pp. 183-188

[4] Hauer J F, Demeure C J and Scharf L L 1990 Initial results in Prony analysis of power system response signals. IEEE Trans. Power Syst. 5: 80-89

[5] Tripathy P, Srivastava S C and Singh S N 2009 An improved Prony method for identifying low frequency oscillations using synchro-phasor measurements. In: Proceedings of the IEEE International Conference on Power Systems (ICPS 2009), Kharagpur, India, pp. 1-5

[6] Rai S, Lalani D, Nayak S K, Jacob T and Tripathy P 2016 Estimation of low-frequency modes in power system using robust modified Prony. IET Gener. Transm. Distrib. 10: 1401-1409

[7] Tripathy P, Srivastava S C and Singh S N 2011 A modified TLS-ESPRIT-based method for low-frequency mode identification in power systems utilizing synchrophasor measurements. IEEE Trans. Power Syst. 26: 719-727

[8] Wies R W, Pierre J W and Trudnowski D J 2003 Use of ARMA block processing for estimating stationary low-frequency electromechanical modes of power systems. IEEE Trans. Power Syst. 18: 167-173

[9] Larsson M and Laila D S 2009 Monitoring of inter-area oscillations under ambient conditions using subspace identification. In: Proceedings of the IEEE PES General Meeting, Calgary, Canada, pp. 1-6 
[10] Ning J, Pan X and Venkatasubramanian V 2013 Oscillation modal analysis from ambient synchrophasor data using distributed frequency domain optimization. IEEE Trans. Power Syst. 28: 1960-1968

[11] Zhang P, Wang X, Wang X and Thorp J S 2014 Synchronized measurement based estimation of inter-area electromechanical modes using the Ibrahim time domain method. Electr. Power Syst. Res. 111: 85-95

[12] Thambirajah J, Thornhill N F and Pal B C 2011 A multivariate approach towards inter-area oscillation damping estimation under ambient conditions via independent component analysis and random decrement. IEEE Trans. Power Syst. 26: 315-322

[13] Turunen J, Rauhala T and Haarla L 2010 Selecting wavelets for damping estimation of ambient-excited electromechanical oscillations. In: Proceedings of the IEEE PES General Meeting, Providence, USA, pp. 1-8

[14] Zhang P, Teng Y, Wang X and Wang X 2015 Estimation of inter-area electromechanical modes during ambient operation of the power systems using the RDT-ITD method. Int. J. Electr. Power Energy Syst. 71: 285-296

[15] Turunen J, Thambirajah J, Larsson M , Pal B C, Thornhill N F, Haarla L C, Hung W W, Carter A M and Rauhala T 2011 Comparison of three electromechanical oscillation damping estimation methods. IEEE Trans. Power Syst. 26: 2398-2407

[16] Seppanen J M, Turunen J, Koivisto M, Kishor N and Haarla L C 2014 Modal analysis of power systems through natural excitation technique. IEEE Trans. Power Syst. 29: 1642-1652

[17] James G H, Carne T G and Lauffer J P 1993 The natural excitation technique for modal parameter extraction from operating wind turbines. SAND 92-1666 UC-261, Sandia National Laboratories

[18] Juang J N and Pappa R S 1985 An eigensystem realization algorithm for modal parameter identification and model reduction. J. Guid. Control Dyn. 8: 620-627

[19] Rai S, Tripathy P and Nayak S K 2015 A nonlinear filtering technique along with RD and TLS-ESPRIT for mode identification of ambient data. In: Proceedings of the Annual IEEE India Conference (INDICON 2015), New Delhi, India, pp. 1-6

[20] Rai S, Tripathy P and Nayak S K 2016 An efficient wavelet based technique for oscillatory mode identification of ambient data via RD and TLS-ESPRIT. In: Proceedings of the National Power Systems Conference (NPSC 2016), Bhubaneswar, India, pp. 1-6

[21] Aharon M, Elad M and Bruckstein A 2006 K-SVD: an algorithm for designing overcomplete dictionaries for sparse representation. IEEE Trans. Signal Process. 54: 4311-4322

[22] Rubinstein R, Bruckstein A M and Elad M 2010 Dictionaries for Sparse Representation modeling. Proc. IEEE 98: $1045-1057$

[23] Kundur P 1994 Power system stability and control. New York, McGrawHill

[24] WECC 2005 Report and data of WECC. Available online at $\mathrm{ftp} / / / \mathrm{ftp}$. bpa.gov/pub/WAMSInformation/

[25] PDCI 2005 PDCI probe testing plan. Available online at http://www.transmission.bpa.gov/business/operations/ SystemNews/

[26] Xiao J, Xie X, Han Y and Wu J 2004 Dynamic tracking of low-frequency oscillations with improved Prony method in wide-area measurement system. In: Proceedings of the IEEE PES General Meeting, Denver, USA, pp. 1104-1109

[27] Tropp J A and Gilbert A C 2007 Signal recovery from random measurements via orthogonal matching pursuit. IEEE Trans. Inf. Theory 53: 4655-4666

[28] Wang J, Kwon S and Shim B 2012 Generalized orthogonal matching pursuit. IEEE Trans. Signal Process. 60: 6202-6216

[29] Rubinstein R, Zibulevsky M and Elad M 2008 Efficient implementation of the K-SVD algorithm and the Batch-OMP method. Technical Report, CS, Technion, Israel

[30] Brincker R, Kren S, Kirkegaard P H and Rytter A 1992 Identification of dynamic properties from correlation function estimates. Bygningsstatiske Meddelelser 63: 1-38

[31] IEEE Std C37.118.1aTM 2014 IEEE Standard for synchrophasor measurements for power systems 